\title{
The Turbo Blade Mode in Different Constrains of Blisk
}

\author{
Yun $\mathrm{He}^{1, \mathrm{a}}$, Song Feng ${ }^{2, \mathrm{~b}}$, Man $\mathrm{Xu}^{3, \mathrm{c}}$ and Zhuan $\mathrm{Li}^{1, \mathrm{~d}}$ \\ ${ }^{1}$ Shaanxi Electronic TECHNICAL COLLEGE, XI'AN 710125, China; \\ ${ }^{2}$ XI'AN INSTITUTE OF OPTICS AND PRECISION MECHANICS, XI'AN 710119, China; \\ ${ }^{3}$ SuZhou Siliyo Science And Technology CO.LTD, SuZhou 215163, China. \\ aheyun1713@163.com, ${ }^{b}$ todaysong1314@163.com, ${ }^{c}$ nancy.xu@yeah.com, ${ }^{\mathrm{d}} 775192135 @ q q . c o m$
}

Keywords: The turbo blade, different constrains, FEM, the modal test.

\begin{abstract}
The blade of the turbo blisk often cracks due to rotor's resonance. The research on influence degree to the turbine blade mode with different constrains of blisk can gain the universal fixture in the modal tests. For this purpose, the vibrant characteristic of blisk was gained by FEM, as well as the modal test of turbine blade was applied in free and intermediate fixed status respectively. The simulation results agreed very well with test ones, which indicated that the influence of blisk constraint can be ignored in modal test when the blisk stiffness is much bigger than the blad.
\end{abstract}

\section{Introduction}

The turbo blisk is a new structural component of aero-engine, which can highly improve the thrust-weight ratio and the engine reliability. Whereas some of the problems come forth in using, such as blades often cracks due to rotor's resonance [1,2]. For the process of changing the blade in blisk is very difficult, the whole blisk is often scrapped even by the reason of only one blade's damage[3].Therefore, turbine blade modal analysis and modal test are necessary in design and retrofit phase to avoid the blade resonance. However, the turbo blade could not be separated with blisk, which leads that the modal test cannot be conducted independently by disassembling the blisk into single blade. What is more, the coupling of the turbine wheel and the different effects on the turbine blade mode with the different restraint state of blisk must be taken into account seriously, although the bending stiffness has little differences with the change of turbine wheel surface's thickness. In addition, the current aviation industry standards are devoid of the relevant criterion to the blisk constrains in the modal test, so the high cost is inevitable to produce a variety of fixtures to match different types of the blisk if according to the actual state of constraints. In this paper, the general rule of turbine blade mode changing with the stiffness of the blisk was studied to solve this problem, and the constraint state having little effect on the mode of turbine blade was proved when the bending stiffness of the blisk was much larger than that of the blades. So based on theoretical and experimental results, the modal test with central fixed constraint was proved to be more reasonable obviously.

\section{The Finite Element Analysis of Blisk}

The section headings are in boldface capital and lowercase letters. Second level headings are typed as part of the succeeding paragraph (like the subsection heading of this paragraph).

For the turbine blisk is cyclic symmetrical, in this paper we only take five pieces of the turbine blades and the relevant fan-shaped portion of the blisk to process the Finite Element Analysis. The Material properties are shown as table $1[4,5]$.

Table 1 Material properties

\begin{tabular}{ccl}
\hline The elastic modulus $(\mathrm{E})$ & Poisson's ratio $(\mu)$ & Density $(\rho)$ \\
\hline 110Gpa & 0.34 & $4650 \mathrm{~kg} / \mathrm{m}^{3}$ \\
\hline
\end{tabular}

And the three-dimensional solid unit is adopted to mesh the overall finite element, the results shown in Figure 1 below. 


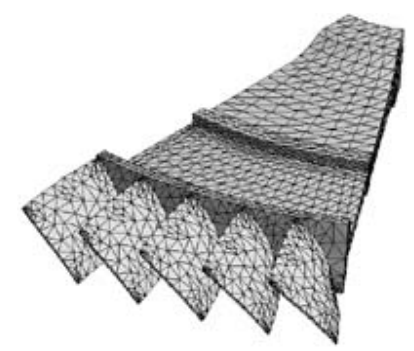

Fig. 1 The finite element model of blisk

Then calculate the finite elements in free and intermediate fixed state separately. Firstly, get the first-three order mode shapes with adding none of constraints on the disk, shown as in Figure 2. Then get the first third-order mode shapes with imposing constraints on the upper and lower ends of the blisk loop surface, shown as in Figure 3[6].

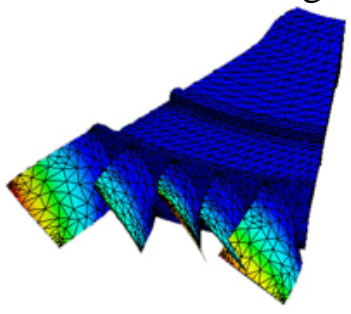

(a) the first order

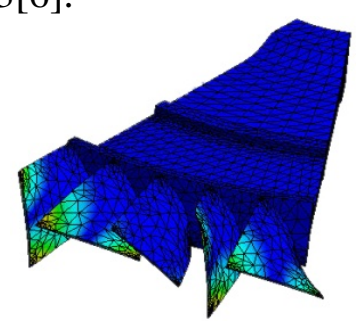

(b) the second order

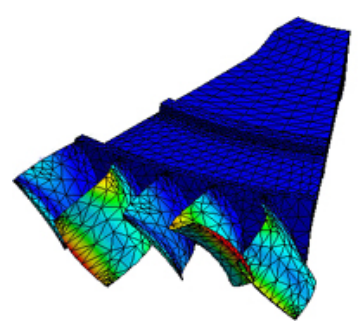

(c) the third order

Fig.2 The first third-order modal shape in the free state

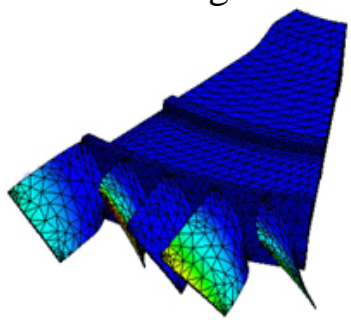

(a) the first order

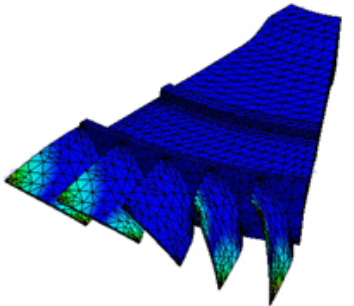

(b) the second order

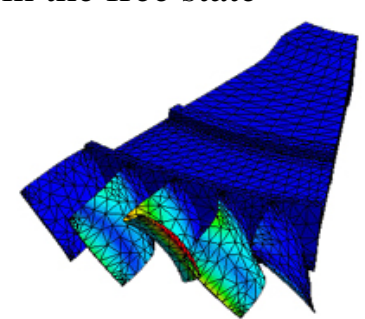

(c) the third order

Fig.3 The first third-order modal shape in intermediate fixed state

And the frequency distribution in the two states is as shown in the following table 2.

Table 2 The frequency distribution in the two states

\begin{tabular}{cccc}
\hline \multirow{2}{*}{ Order } & $\begin{array}{c}\text { Modal frequency } \\
\text { in free state }(\mathrm{Hz})\end{array}$ & $\begin{array}{c}\text { Modal frequency } \\
\text { intermediate fixed state }(\mathrm{Hz})\end{array}$ & Modal shape description \\
\hline 1 & $828.87 \sim 874.46$ & $829 \sim 856.96$ & bending vibration \\
2 & $2232 \sim 2258$ & $2232.7 \sim 2258.5$ & torsional vibration \\
3 & $2998 \sim 3354$ & $3055 \sim 3368.2$ & bending vibration \\
\hline
\end{tabular}

As can be seen from the table 2 and the Figure 2-3, the first-three order modal frequency distribution in the two states is very close, and the mode of vibration in the two states matches very well. Obviously the different blisk constrains have little effect on the turbine blade mode .

However the first-three order modal frequencies show zonal distribution, and the values float around. Because the turbine blades are connected to the blisk body by hoop, while the hoop cannot be completely rigid, that would lead to elastic coupling between the blades and the blisk body. If impose constraints on the upper and lower ends to ensure zero displacement to further reduce the coupling , and calculate the first-three order modal frequency distribution shown as table 3.

Table 3 The frequency distribution when further reduce the coupling

\begin{tabular}{cc}
\hline Order & Modal frequency when further reduce the coupling $(\mathrm{Hz})$ \\
\hline 1 & $862 \sim 864$ \\
2 & $2247 \sim 2251$ \\
3 & $3224 \sim 3229.7$ \\
\hline
\end{tabular}

Apparently the modal frequency distribution tends to a fixed value when the blisk hoop is fixed, which is more closer to the true value of blade frequency. 
For the swing of blisk body is almost zero seen from Figure 2,3 ,which indicates the coupling effects little and the first-three order modal frequency distributions in free and intermediate fixed state are all closed to the real frequency distribution of blades. What's more, the bending rigidity of blisk is about 78 times larger than that of the blade by calculating the blade and the blisk separately (where the bending stiffness of the blade is $4.8 \times 10^{9} \mathrm{~N} / \mathrm{m}$, that of blisk is $3.74 \times 10^{11} \mathrm{~N} / \mathrm{m}$ ), which is the just reason that the coupling exists, but effects little.

\section{Modal test for the blisk system}

Analysis the Modal tests results in free and intermediate fixed state separately [7, 8].

Place the blisk on the sponge pad to simulate the free status. For the natural frequency of the vibration system consisting of the blisk and the sponge pad is less than $10 \mathrm{~Hz}$, far down from the first-order natural frequency of the blisk which is $253.3 \mathrm{~Hz}$.

Connect the center hole of blisk to the big rigid mass of about $500 \mathrm{~kg}$ by two mass blocks to simulate the intermediate fixed constraints. The modal test results in free and intermediate fixed states were shown as Table 4 and Fig. 4 respectively.

Table 4 The modal test results in the free and Intermediate fixed states

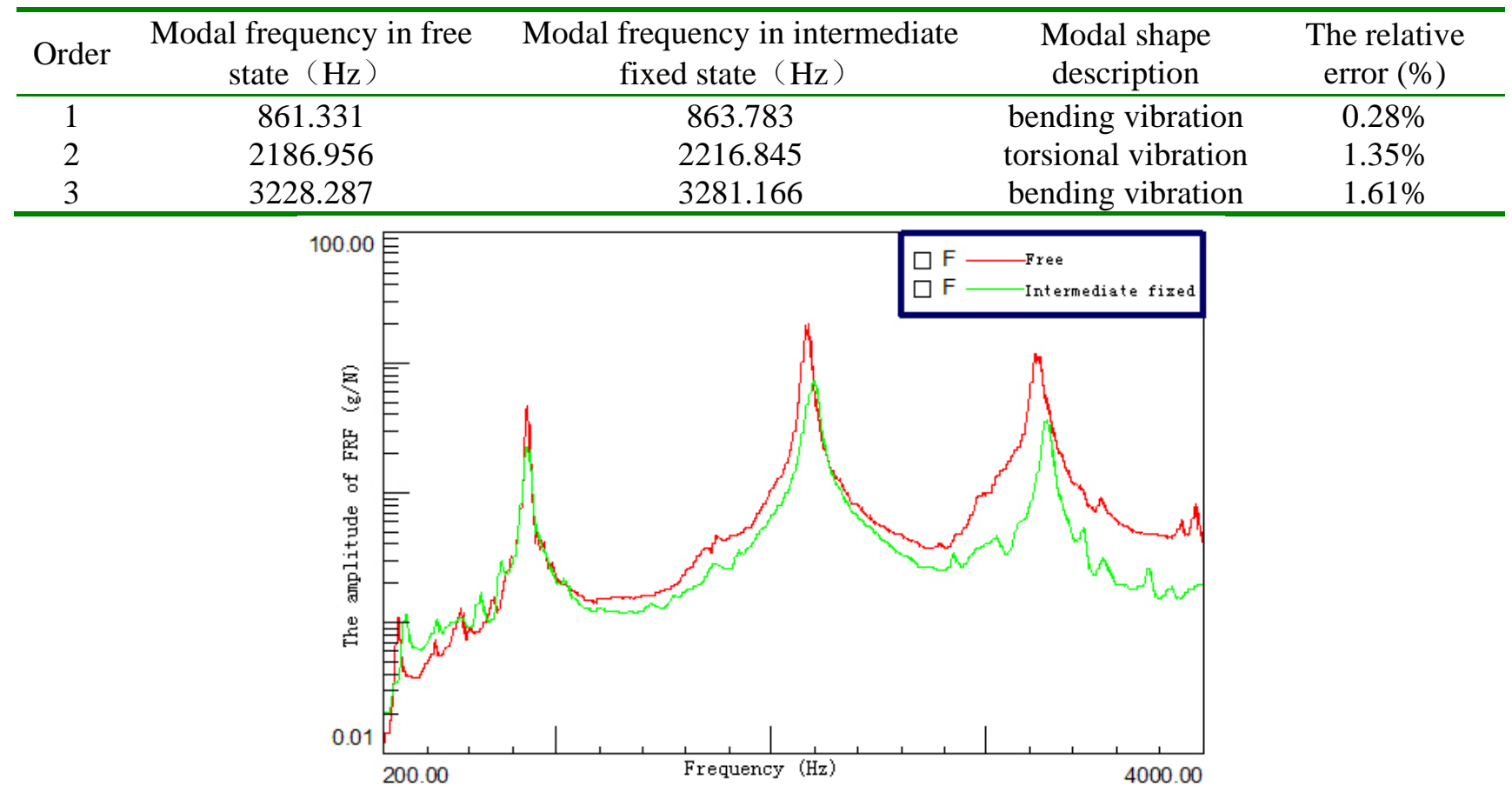

Fig. 4 The FRF of the blisk in the two states

The test conditions and the analytic technique in the two states were nearly the same, and the blades were chosen diversely in the two tests to make the test results more universal. Taking into account each blade itself has a certain frequency dispersion, and the error is within the allowable range, which further proves the impact of different blisk constraints on first-three natural frequencies and mode shapes is too small to be ignored.

As can be seen from figure4, the first-three modal frequencies of the two states are basic coincident, and the nature frequencies of the blisk itself only appears in the low frequency band with small peak, having nearly no influence on blades frequencies. That indicates that the coupling affects little, and the modal parameters of blades are relatively independent, which is also consistent with the finite element analysis results. 


\section{Comparison between Simulation and Tests}

Contrast the FEM results to the modal test results in the two states as shown in Table 5 and 6. Table5 Contrast simulation and test results in free status

\begin{tabular}{cccc}
\hline Order & The simulation results $(\mathrm{Hz})$ & The modal test results $(\mathrm{Hz})$ & The maximum error (\%) \\
\hline 1 & $828.87-874.46$ & 861.331 & $3.8 \%$ \\
2 & $2232-2258.5$ & 2186.956 & $3.3 \%$ \\
3 & $2998-3354$ & 3228.287 & $7.1 \%$ \\
\hline
\end{tabular}

Table 6 Contrast simulation and test results in intermediate fixed status

\begin{tabular}{cccc}
\hline Order & The simulation results (Hz) & The modal test results (Hz) & The maximum error (\%) \\
\hline 1 & $829-856.96$ & 863.783 & $4 \%$ \\
2 & $2232.7-2258.5$ & 2216.845 & $1.9 \%$ \\
3 & $3055-3368.2$ & 3281.166 & $6.7 \%$ \\
\hline
\end{tabular}

The maximum error chose the modal test results as a benchmark. As can be seen from the tables, the simulation results and the modal test results are basically consistent. The maximum error of higher order modes is greater, mainly because the number of finite elements is always limited, impossible to be infinite, which affects the calculation accuracy of higher order modes. While the maximum error is still within $8 \%$, which can meet project requirements well and is still credible.

\section{Conclusions}

This paper studied the influence degree to the turbine blade mode with different constrains of blisk. Though the finite element analysis of blisk, the elastic coupling between the blades and the blisk body is proved to be inevitable just because the blisk hoop exists, which lead the first-three order modal frequencies of blisk show zonal distribution, and the values float around. While the coupling effect is very little, because the blisk body's amplitudes of the first-three order modal shapes is almost zero. And the modal frequency distribution is also proved to tend to a fixed value very close to the blade's nature frequency when the blisk hoop is fixed. Analysis the Modal tests results in free and intermediate fixed state and conclude that the first-three modal frequencies in the two states are basic coincident and are far away from the effect band of the blisk body's nature frequencies. At last, the simulation results and the modal test results can be found to be basically consistent by comparison.

In brief, the different constrains of blisk are identified to have little effect to turbine blade mode, when the bending stiffness of blisk is more than 70 times higher than that of blade. And at that time, the fixture of the blisk system can be unfiled and simplified to adopt the intermediate fixed constrains, which comes to be a good choice to cost little, have smaller error, and be more easier to implement.

\section{References}

[1]. Andersson S,trollheden. Aerodynamic design and development of a two-stage supersonic turbine for rochet engines. AIAA 1999-2 192.

[2]. Whlen U, Sjunnesson I, Groth P. Measurement and prediction of unsteady blade loads in a supersonic/transonic turbine.AIAA 97-2 755.

[3]. CHEN GUANG. Application of blisk in the foreign aeroengine[J]. Aeroengine,1999,25(I):1-6.

[4]. FAN Zhiqiang, MA Mei, WANG Rongqiao. The optimization of the blisk in aeroengine[J].Gas Turbine Experiment and Research,2000,13(4):27-30. (In Chinese)

[5]. LI Lunwei , LU Shan. Structure optimiazation design techniques for multi-web fan disk based on ANSYS platform[J]. Journal of Aerospace power,2011,26(10):2245-2250. (In Chinese)

[6]. GU SongNian, et al. Modal parameter identification of structure under base exitation. Journal of Mechanical Strength, 1999, 21(4):244 288(In Chinese) 
[7]. LI Shu-ming, HUANG Yan-xiao, CHENG Guan-bing. Inherent Modal Analysis of Aero-Engine Turbine Disc. JOURNAL OF CIVIL AVIATION UNIVERSITY OF CHINA. 2008, 26(2):8 11.

[8]. ZHAO Jian, YU Ming. Vibrational and Acoustic Modal Analyses of Gas Turbine Disk in Aeroengine. ACTA ARMAM ENTARII. 2010,31(1):179 183. 